\title{
NDT of Layered Structures Using Pulse-Thermography and THz-TDS Imaging
}

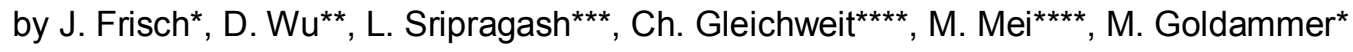 \\ *Siemens AG, CT RDA IOT DPR-DE, Otto-Hahn-Ring 6, 81739 München, Germany, julia.frisch@siemens.com, \\ matthias.goldammer@siemens.com \\ ${ }^{* *}$ Munich University of Applied Sciences, Department of Applied Sciences and Mechatronics, Lothstraße 34, \\ 80335 München, Germany, datong.wu@hm.edu \\ ***Siemens Inc., CT REE MDM CMI-US, 5101 Westinghouse Boulevard, 28273-9640 Charlotte, NC, USA, \\ letchuman.sripragash@siemens.com

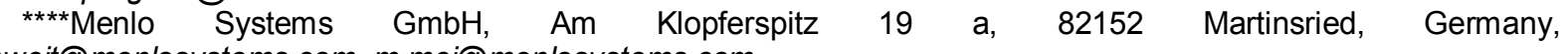

c.gleichweit@menlosystems.com, m.mei@menlosystems.com

\begin{abstract}
The nondestructive testing (NDT) techniques active thermography and terahertz time domain spectroscopy (THz-TDS) are both newcomers to the large range of established NDT methods: Both are contactless imaging techniques and well suited for analysing layered materials e.g. ceramic coatings, polymer laminates and glued materials. These two methods are compared for characterising ceramic coatings. Measurement results show these two methods are practicable and complimentary for NDT applications of layered structures. The pulse-thermography is suitable for fast screening inspection and THz-TDS imaging performs well for the detailed investigation of coatings and boundary variations.
\end{abstract}

\section{Introduction}

Today, gas turbines strive to be ever more efficient. One way to achieve a higher efficiency is to increase the temperature of the gas. However, the higher the temperature, the higher the wear of the turbine parts resulting in declined component life. Plus, even the employed super alloys reach their limit and their melting point at $1100^{\circ} \mathrm{C}$ to $1200^{\circ} \mathrm{C}$. To withstand the high temperatures the super alloy blades are coated with a thermal barrier coating (TBC). For one thing, the TBC acts as a thermal shield keeping a lower temperature at the base material through the insulating functionality of its porous structure. For another, it acts as a barrier to oxidation of the base material. The thickness of the TBC varies from a hundred microns to millimetres with the position of the coated part in the gas turbine. Furthermore, the thickness is strongly dependent on the coating process.

One of the most commonly used ceramic TBC materials is yttria-stabilised zirconia $\left(\mathrm{YSZ}, \mathrm{Y}_{2} \mathrm{O}_{3}+\mathrm{ZrO}_{2}\right)$. After first administering a metallic bond coat (BC) for further corrosion protection and better ceramic growth, the YSZ is deposited on top. There are two main coating processes: air plasma spray and electron beam physical vapour deposition. Today, mostly electron beam physical vapour deposition is used because it can be applied more locally. One problem is that the process is hard to control and coordinate and hence the deposited thickness and thermal conductivity are also hard to control precisely. However, it is important to know the coating thickness, the porosity and the thermal conductivity to know the properties of a turbine blade and to hint at its lifetime.

Pulse-thermography or flash thermography is one of the newer NDT techniques. Yet, it is the standard technique when it comes to examining the coating of turbine blades for delaminations or the cooling air conducts for blockage or rather permeability. Even though it has the advantage over techniques such as ultrasonic and eddy current testing by being non-contact, imaging i.e. two-dimensional and already in use on every turbine blade, it is almost never employed to evaluate TBC coating thickness or thermal conductivity. In comparison to other thermal techniques such as the flash method [1, 2], the phase of photothermal emission analysis method [3], or the laser flash method [4], the method of flash thermography employed for these experiments needs neither two-sided access nor a strong laser for excitation.

In 1986, Balageas et al. [5] developed the first set of analytical solutions for layered materials. These were the basis on which Sun [6] created a numerical model using the finite difference solution technique including volume heating and flash duration effects. In this work, a numerical model using finite difference solution [7] is also applied to the experimental data to extract the important material parameters of coating layer thickness and thermal conductivity via a nonlinear regression fit.

Due to real-time, non-contact and spectroscopic measurements, the $\mathrm{THz}$ technology has become one of the promising future NDT methods [8, 9]. With a 3D scanning device, the depth-resolved imaging of a specimen is possible. Compared to X-rays, ultrasound, microwaves, and thermography, $\mathrm{THz}$ signals provide material information in the $\mathrm{THz}$ wave range. Since $\mathrm{THz}$ radiation can penetrate through non-metal materials, the technology is suitable for molecular materials such as ceramics, semiconductors, fibre composites and polymer foams. In recent years, THz technology has been increasingly used for NDT of glass fibre reinforced plastics (GFRP), foams and other composites [10, 11]. As a NDT method, the THz signal can be used to measure thickness of layers and to detect bonding defects between layers or between a coating layer and the substrate. 
An important field of application is the non-destructive and non-contact testing of ceramic coatings. The $\mathrm{THz}$ Time-Domain systems allow for simultaneous measurement of the coating related parameters and the $\mathrm{THz}$ spectrum of the coating.

\section{Experimental}

In the pulse-thermography experiments, a FLIR SC 7600 (FLIR Systems, Inc., Portland OR, USA) in quarter frame mode with $160 \times 128$ pixels, an integration time of $2000 \mu \mathrm{s}$ and a frame rate of $469 \mathrm{~Hz}$ was employed. The camera consists of an InSb-detector and a Stirling cooler. With the mid-wave broad band object lens, the band from 1.5 to $5 \mu \mathrm{m}$ can be covered with a NETD of $25 \mathrm{mK}$. The camera was not calibrated and the arbitrary intensity values were used in the analysis. The pulse-thermography was performed in reflection with both, the camera as well as the flash, on the zirconia coated side of the sample.

For the pulsed excitation, a Broncolor Grafit A4 (Bron Elektronik AG, Allschwil, Switzerland) photographic flash with a maximum of $3.2 \mathrm{~kJ}$ was operated at $1054 \mathrm{~J}$ with a flash duration of $1 / 750 \mathrm{~s}$. Characteristic for this flash is that the flash itself is cut off after exactly the set time. Furthermore a PMMA filter was employed.

For the eddy current thickness measurements, a FISCHER DUALSCOPE MPOD (Helmut Fischer GmbH, Sindelfingen, Germany) was used.

For the THz-TDS experiments, the terahertz measurement system TERA ASOPS (Menlo Systems GmbH, Martinsried, Germany) was applied. It is based on two C-fiber femtosecond fibre lasers with a repetition rate of $250 \mathrm{MHz}$ and $90 \mathrm{fs}$ pulses at $1560 \mathrm{~nm}$ central wavelength. Two fibre coupled antennas of type TERA15-FC were used (the emitter TERA15-TX-FC and the receiver TERA15-RX-FC). The terahertz emitter and receiver are based on the principle of a photoconductive switch, which generates pulses with up to $5 \mathrm{THz}$ bandwidth and a continuous power of about $40 \mu \mathrm{W}$. The optical power of the fibre lasers is about $30 \mathrm{~mW}$ with a pulse energy of about $0.3 \mathrm{~nJ}$ (corresponding to $5 \times 10^{-13} \mathrm{~J}$ for the $\mathrm{THz}$ radiation). The focus of the used lenses (TPX35) is approximately $1 \mathrm{~mm}$ (full bandwidth, maximum at $0.3 \mathrm{THz}$ ) and $0.2 \mathrm{~mm}$ at high frequencies (about $2 \mathrm{THz}$ ). The repetition rates of both lasers were locked and stabilised by using two repetition rate synchronisation electronic devices (RRE SYNCRO). A 16-bit 10-MHz data acquisition board is used in the scan control program of Menlo Systems. The measurement speed can be adjusted in the measurement system. For the measurements, the scan speed was $50 \mathrm{~Hz}$, which means 50 time-domain traces per second, therefore also 50 spectra per second.

\section{Zirconia thermal barrier coatings on Inconel 738}

For these experiments, a nickel-based super alloy sample coated with a layered TBC-system was examined. The substrate material is Inconel 738 (IN 738). It was coated first with a metallic bond coat of a thickness between 100 and $230 \mu \mathrm{m}$. Afterwards, YSZ was added on top by electron beam physical vapour deposition.

Table 1. Nominal, calculated and measured thickness values for the different layers of the zirconia coated IN 738 sample.

\begin{tabular}{|l|r|r|r|r|}
\hline & \multicolumn{1}{|c|}{ Step 1 } & \multicolumn{1}{|c|}{ Step 2 } & \multicolumn{1}{|c|}{ Step 3 } & \multicolumn{1}{|c|}{ Step 4 } \\
\hline Nominal TBC thickness $(\mathbf{m i l s})$ & 6.0 & 7.0 & 9.0 & 11.0 \\
\hline Nominal TBC thickness $(\boldsymbol{\mu m})$ & 152.4 & 177.8 & 228.6 & 279.4 \\
\hline $\begin{array}{l}\text { Substrate thickness measured } \\
\text { with calliper }(\mathbf{m m})\end{array}$ & 8.85 & 8.85 & 8.85 & 8.85 \\
\hline $\begin{array}{l}\text { Sample thickness measured } \\
\text { with calliper }(\mathbf{m m})\end{array}$ & 9.30 & 9.29 & 9.43 & 9.37 \\
\hline Calculated BC thickness $(\boldsymbol{\mu m})$ & 200.7 & 175.9 & 229.0 & 128.4 \\
\hline
\end{tabular}

There are four steps of different TBC thickness to the sample, cf. Table 1 and Fig. 1. As mentioned before, TBC coating process is hard to control and therefore the actual thickness differs from the nominal TBC thickness value in Table 1. The also included BC thickness was calculated by subtracting the TBC thickness measured by eddy current and the substrate thickness from the sample thickness.

Due to YSZs partial transparency in the mid-wave infrared band and its low emissivity, one half of the sample was additionally sprayed with graphite absorbing coating (Graphit 33, Kontakt Chemie). While this graphite layer has almost no impact on the properties of the sample measured by eddy current or pulse-thermography [12], there is a strong influence on the measurement with THz-TDS imaging due to the high refractive index of graphite. The graphite layer causes an additional time difference of around $1 \mathrm{ps}$. Therefore, the pulse-thermography measurements are performed on the graphite coated upper half of the zirconia coated IN 738 sample, whereas the lower half without graphite is used for the THz measurements. 


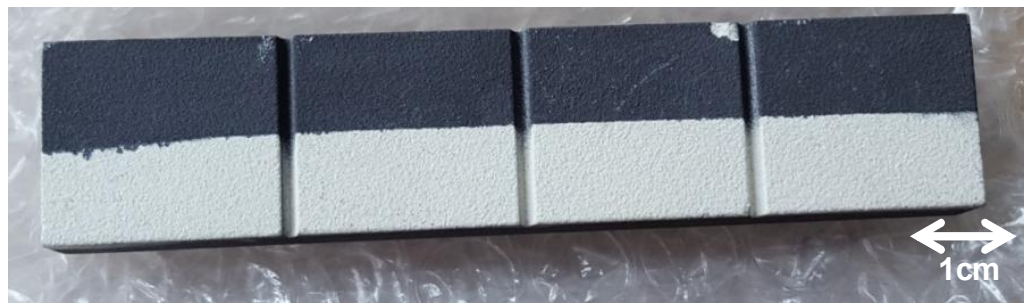

Fig. 1. Photo of zirconia coated IN 738 sample. Dimensions: $89 \mathrm{~mm} \times 19 \mathrm{~mm} \times 9 \mathrm{~mm}$. Upper half is additionally sprayed with graphite.

\section{Pulse-Thermography}

Pulse-thermography is the act of heating a sample surface with a short light pulse and thereafter examining the transient sample surface temperature with an IR camera. Fig. 2 corresponds to the thermogram of the zirconia coated IN 738 sample directly after flash excitation. The time evolution of this surface temperature which depends on the heat diffusion process then provides information about the thermophysical properties, the inner composition such as layer structure, and subsurface features such as delaminations. The absorption coefficient of the sample surface and the effusivity of the top layer govern the heating of the surface from the absorbed flash and therefore the recorded IR signal. As the flash system allows cutting off the flash duration, the flash can be treated as instantaneous for the purpose of the measurements presented here. After the flash, the signal is determined by the heat conduction in the layered system driven by the thermal parameters and the layer geometry $[13,14,15]$. When using a flash system without the capability to limit the flash duration, the finite duration has to be taken into account as most flashes have, after a fast intensity rise, an exponential intensity decay due to the discharging of the capacitor.

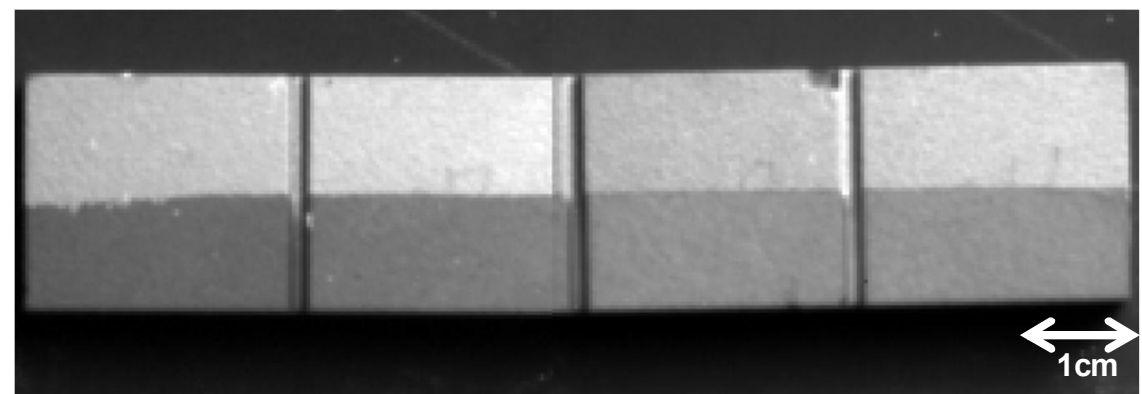

Fig. 2. Thermogram of zirconia coated IN 738 sample directly after flash excitation with subtracted background. The figure consists of two partial images due to the quarter frame mode of the camera.

\subsection{Theoretical background of heat diffusion}

The one-dimensional Fourier heat equation in Eq. (1) describes the heat diffusion process for a onedimensional system. It includes the thermal conductivity $k$, the absolute temperature $T$, the body heat generation rate per volume $\dot{q}$, the density $\rho$, the specific heat capacity $c$ and the time $t$.

$$
k \frac{d^{2} T}{d z^{2}}+\dot{q}=\rho c \frac{d T}{d t}
$$

The flash used in pulse-thermography usually heats a large area of the sample uniformly in the $x-y-p l a n e$. Hence, the heat transfer over the entire sample can be considered as one-dimensional in the depth- or z-direction and the data analysis can be performed in 1D. Furthermore, the analysis is valid for ideal flash conditions only. This means instantaneous flash energy absorption at the surface as well as a very thick substrate in respect to the coating thickness and no concern is given to finite flash duration and TBC translucency.

Balageas et al. [5] provided the analytical solutions for layered systems with different heating pulses at the surface. The problem is that with complex layered systems and accounting for finite flash duration, the heat equation in Eq. (1) becomes increasingly difficult to solve analytically. Therefore, a numerical approach which limits computational time is advantageous.

\subsection{Numerical modelling}

To solve the Fourier heat equation in Eq. (1) with the finite difference solution technique, the Crank-Nicholson algorithm (see for example [16]) is applied. The actual discretisation results from looking at the six states or rather the three states $(i-1, i, i+1)$ for each of the two time steps $(j, j+1)$. This results in Eqs. (2) or (3) with $\lambda=\alpha \Delta t / \Delta z^{2}$, $\alpha=k / \rho c$ and the heat input $Q_{i j}=Q \delta\left(z_{i}\right)[6]$. 


$$
\begin{gathered}
k\left\{\frac{\left(T_{i+1}^{j+1}-2 T_{i}^{j+1}+T_{i-1}^{j+1}\right)+\left(T_{i+1}^{j}-2 T_{i}^{j}+T_{i-1}^{j}\right)}{2 \Delta z^{2}}\right\}+Q_{i j}=\rho c \frac{\left(T_{i}^{j+1}-2 T_{i}^{j}\right)}{\Delta t} \\
-\lambda T_{i-1}^{j+1}+2(1+\lambda) T_{i}^{j+1}-\lambda T_{i+1}^{j+1}=\lambda T_{i-1}^{j}-2(\lambda-1) T_{i}^{j}+\lambda T_{i+1}^{j}+Q_{i j}
\end{gathered}
$$

Wherein $Q_{i j}$ can incorporate volume heating and flash duration effects. Considering $m-2$ time steps returns $m-2$ equations for $m$ unknowns which leads to the need for the two boundary conditions for the top and the bottom surface. The heat input on the top surface has already been included in the general heat equation and the bottom surface is considered adiabatic, therefore the two conditions can be written as Eq. (4) and (5) [7].

$$
\begin{gathered}
T_{2}^{j}=T_{1}^{j} \\
T_{n}^{j}=T_{n-1}^{j}
\end{gathered}
$$

For an interface between layer $a$ and layer $b$ in multi-layered materials with negligible interface resistance, Eq. (6) follows as the discretised equation with the spatial grid size $\Delta z$ [7].

$$
-\Delta z k_{a} T_{i-1}^{j}+\left(\Delta z k_{a}+\Delta z k_{b}\right) T_{i}^{j}-\Delta z k_{b} T_{i+1}^{j}=0
$$

In these experiments, the experimental data was fitted with the numerical solution for a three-layer system, consisting of the TBC, the metallic bond coat (BC) and the substrate, using a nonlinear regression method. Since there is no calibration for the camera and the flash duration effect is not considered, the fit of the normalised intensity does not work well due to a big offset in the logarithmic intensity. Therefore, for further evaluation, the second derivative of the logarithmised temperature or in this case the arbitrary intensity in Eq. (7) is used, with $\Delta T=T(t)-T_{0}$. Shepard et al. [17] verified that the second logarithmic derivative is very effective in defect depth characterisation which is analogous to coating thickness measurements.

$$
2^{\text {nd }} \text { Derivative }=\frac{d^{2}(\log \Delta T)}{d(\log t)^{2}}
$$

This method of regression fitting the numerical data to the experimental values can determine three values at once: $Q, e_{1}=\sqrt{k_{1} \rho_{1} c_{1}}$ and $\eta_{1}=L_{1} / \sqrt{\alpha_{1}}$. Only $e_{1}$ and $\eta_{1}$ are parameters of the TBC layer. This leads to the conclusion that of the three important TBC parameters thickness $L$, thermal conductivity $k$ and heat capacity $\rho c$ only two can be determined independently by pulse-thermography [6]. For the fit in this work, the parameters thickness and thermal conductivity were chosen as free fit parameters. Every other parameter was kept at the constant input value.

\subsection{Experimental results}

For a homogenous, non-layered sample of finite thickness, the surface temperature decay after the pulsed excitation is directly proportional to $1 / \sqrt{t}$ until the back wall i.e. a constant value is reached [5]. This leads to a straight line with a slope of -0.5 plotted on a double logarithmic scale. For a layered sample, e.g. a coating on metal, the surface temperature decay is more complex: after reaching the interface between the different layers, the slope of the temperature evolution changes dependent on the quality of the interface and the thermal properties of the layers [5]. The inflection point as well as the new curvature of the curve is determined by the thickness and the thermal properties of the coating layer.

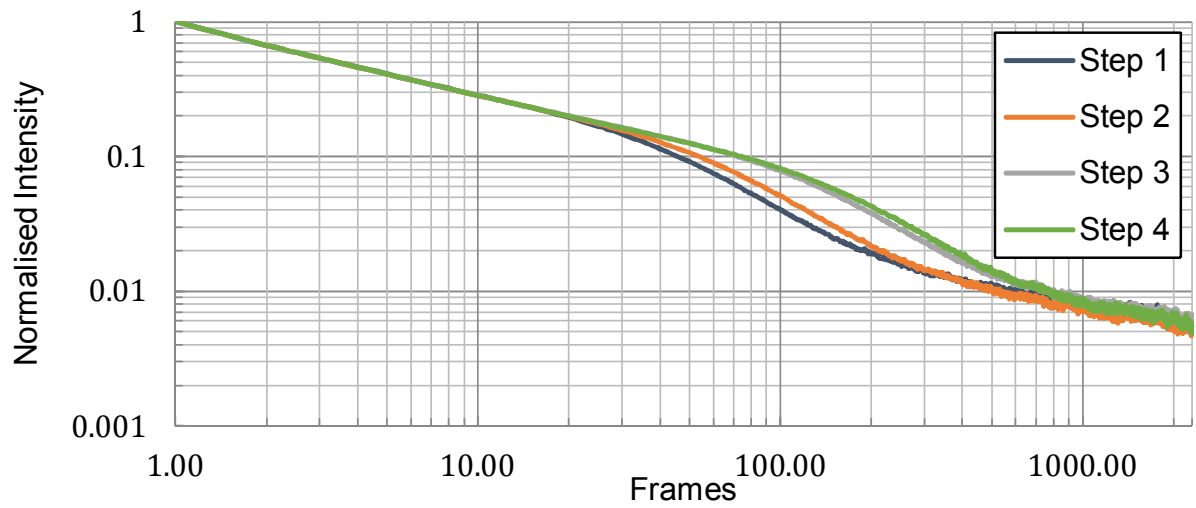

Fig. 3. Normalised intensity evolution of the zirconia coated IN 738 sample after flash excitation. One frame corresponds to 2.13 seconds.

Fig. 3 shows the experimental data in a double logarithmic graph for the four steps of the zirconia coated IN 738 sample. The inflection point of the curves moves to the right, i.e. to later times, for a higher coating thickness. This correlates well with the notion that it takes longer for the heat to reach the interface to the metallic substrate if the coating is thicker. Also, the slope of the curves steepens after the inflection point due to the higher thermal conductivity of the metal in comparison with the ceramic coating. 
The second logarithmic derivative of the experimental data for the four steps of the zirconia coated IN 738 sample is shown in Fig. 4 as continuous lines. For each step only one point on the sample was selected for this figure. Noticeable is again the shift of the minimum to the right for thicker coatings.

Also included in Fig. 4 as dashed lines is the numerical model which was fitted via nonlinear regression to the minima of the experimental data. Basis for the fit is a three-layered system consisting of TBC, BC and the substrate.

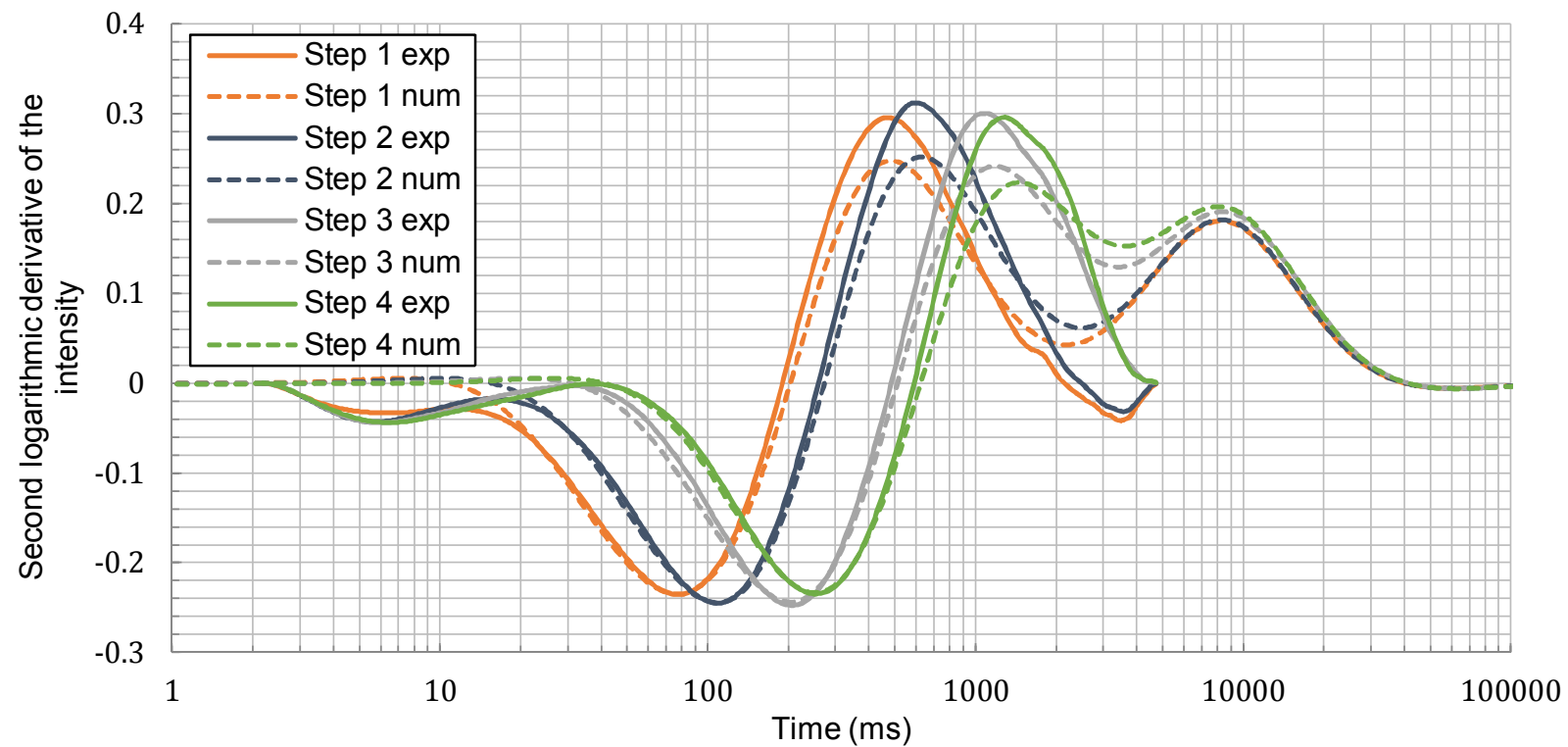

Fig. 4. Experimental (continuous line) and numerical (dashed line) evolution of the second derivative of the logarithmised intensity. The numerical model was fitted to the experimental data via nonlinear regression with the two free parameters TBC thickness and thermal conductivity.

For the fit, the free fit parameters thermal conductivity and coating thickness were chosen. The extracted values are compiled in Table 2 with the values of the standard deviation of the averaging. No error consideration was performed. Table 3 includes all the input material properties for the numerical model.

Table 2. Nominal and extracted values for the YSZ-layer with pulse-thermography. For the error margins the standard deviation of the averaged values was used.

\begin{tabular}{|l|l|l|l|l|}
\hline & Step 1 & Step 2 & Step 3 & Step 4 \\
\hline Nominal TBC thickness $(\boldsymbol{\mu m})$ & 152.4 & 177.8 & 228.6 & 279.4 \\
\hline $\begin{array}{l}\text { Averaged TBC thickness }(\boldsymbol{\mu m}) \\
\text { with numerical flash model }\end{array}$ & $116.3 \pm 2.5$ & $145.3 \pm 3.4$ & $221.9 \pm 7.7$ & $274.8 \pm 2.9$ \\
\hline $\begin{array}{l}\text { Averaged TBC thermal } \\
\text { conductivity }(\mathbf{W} /(\mathrm{mK})) \\
\text { with numerical flash model }\end{array}$ & $0.331 \pm 0.011$ & $0.370 \pm 0.007$ & $0.443 \pm 0.034$ & $0.546 \pm 0.021$ \\
\hline
\end{tabular}

Table 3. Parameters of the different layers of the zirconia coated IN 738 sample (TBC, BC and substrate). Values were either estimated and validated with the model or ${ }^{1)}$ from [18].

\begin{tabular}{|c|c|c|c|}
\hline & $\begin{array}{l}\text { Density } \\
\left(1 \mathrm{e}-3 \mathrm{~kg} / \mathrm{m}^{3}\right)\end{array}$ & $\begin{array}{l}\text { Heat capacity } \\
(1 \mathrm{e}-2 \mathrm{~J} /(\mathrm{kgK}))\end{array}$ & $\begin{array}{l}\text { Thermal conductivity } \\
(\mathrm{W} /(\mathrm{mK}))\end{array}$ \\
\hline TBC & 6.05 & 5.37 & - \\
\hline BC & 7.00 & 4.80 & 5.0 \\
\hline Substrate & $8.11^{1)}$ & $4.19^{1)}$ & 10.0 \\
\hline
\end{tabular}

\section{THz-TDS imaging}

$\mathrm{THz}$ waves are electromagnetic radiation in the frequency range of $100 \mathrm{GHz}$ to $10 \mathrm{THz}$, and are located between infrared radiation and microwaves on the spectrum. This spectral range is an important transition area between photonics and electronics. There are two important applications for $\mathrm{THz}$ technology: $\mathrm{THz}$ spectroscopy and $\mathrm{THz}$ imaging $[19,20]$. Compared to electromagnetic waves in other spectral ranges, $\mathrm{THz}$ radiation has the following application relevant properties: low radiant energy, permeation into many materials (plastics, ceramics, semiconductors, etc.), broadband, coherence, and spectrum fingerprinting for many materials. $\mathrm{THz}$ technology plays an important role in 
modern physics; from Fourier transform infrared spectroscopy to X-ray technology. The THz-TDS finds many applications in material characterisation and quality control due to simultaneous determination of time and spectrum properties of materials (Fig. 5) [21, 22].
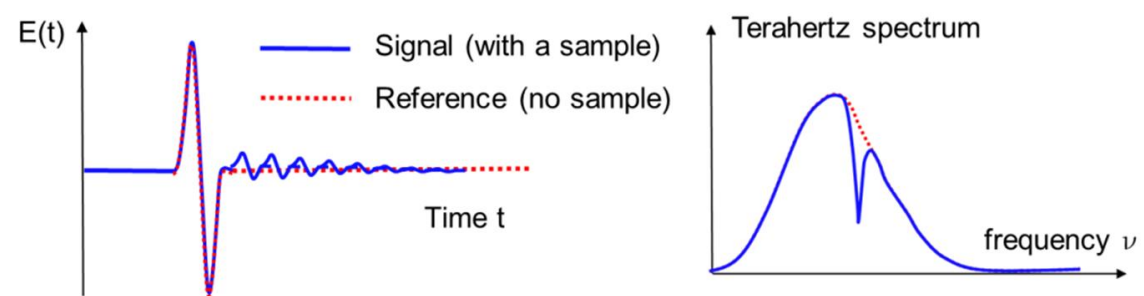

Fig. 5. Schematic temporal terahertz time domain spectroscopy (THz-TDS) signal (left) and calculated THz absorption spectrum by means of the Fourier transform.

\subsection{Measurement principle} 6) [23].

In the THz-TDS measuring system, two femtosecond lasers are used to generate and detect THz pulses (Fig.
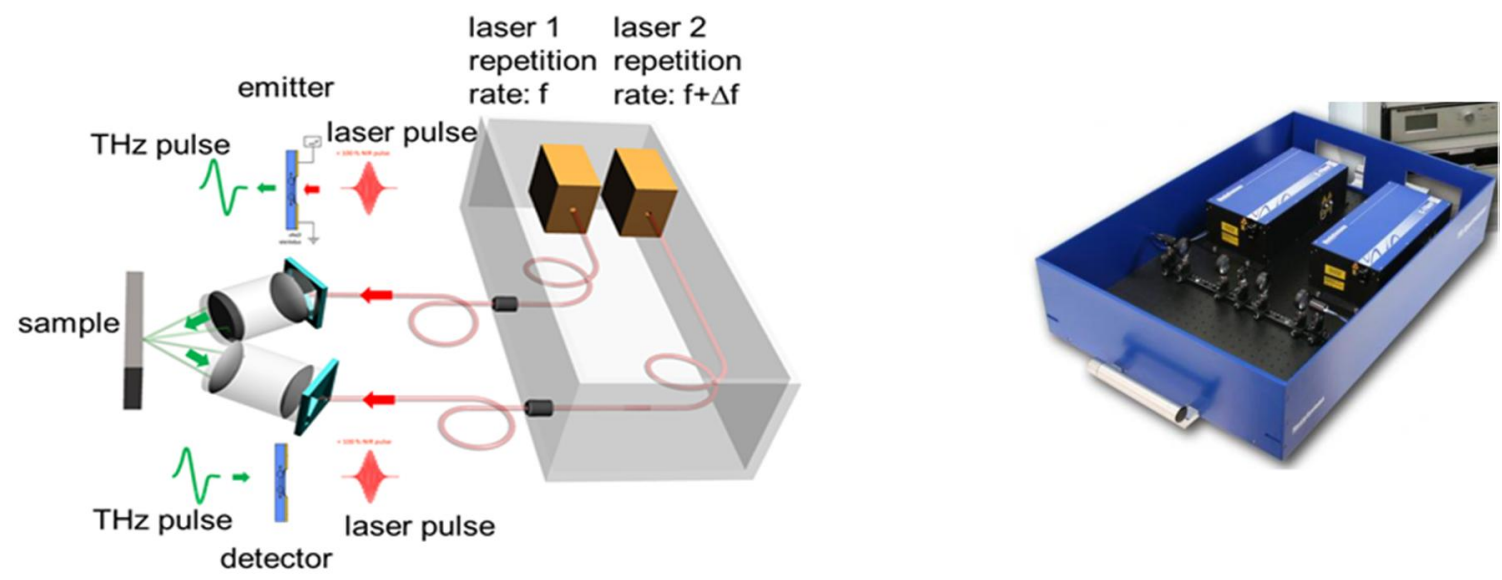

Fig. 6. Setup of the THz-TDS measuring system based on two femtosecond lasers (left) and the Menlo-THz system (right) [23, 24].

A photoconductive element converts the laser pulse energy into the $\mathrm{THz}$ pulses and another photoconductive element operates as a receiver. The measuring principle is based on the pump-probe method, in which the second laser is responsible for the sampling of the THz pulses (Asynchronous Optical Sampling System ASOPS).

The two femtosecond lasers are synchronised via radio-frequency locking electronics. The ASOPS principle is shown in Fig. 7, where the sampling pulses are delayed by a time difference of several times $\Delta t$ from the pump laser pulses.

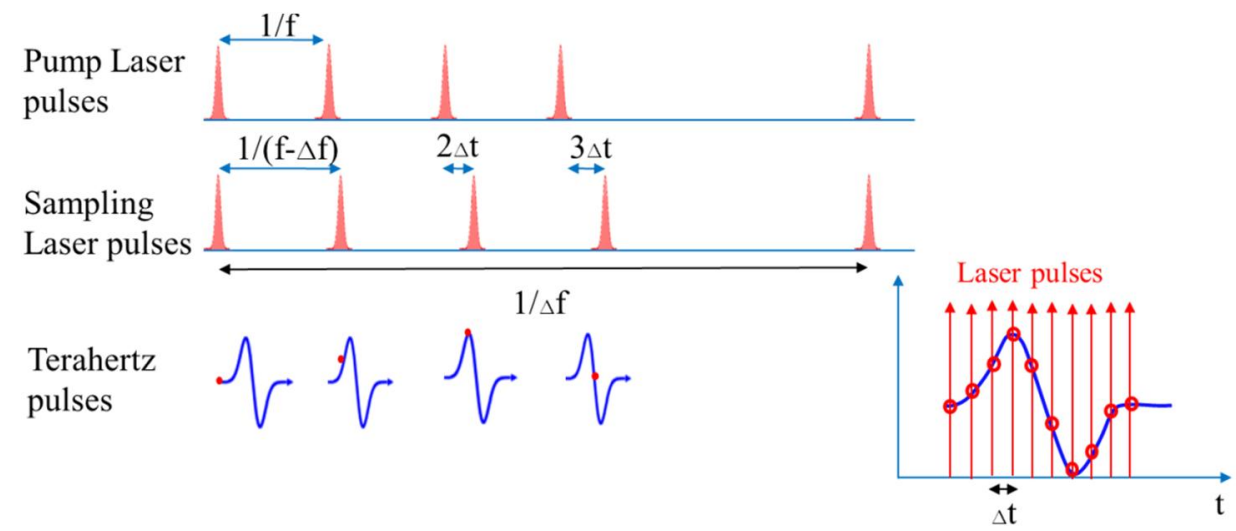

Fig. 7. Asynchronous Optical Sampling System (ASOPS) with one pump laser for THz pulse generation and a second laser for $\mathrm{THz}$ pulse sampling [25]. 
The schematic drawing (Fig. 8) shows the measurement principle of the THz-TDS system. The THz incident pulses are reflected on the interfaces due to changes of layer refractive indices. The polarity of the $\mathrm{THz}$ reflection pulses can vary depending on the sign of the reflectivity. The $\mathrm{THz}$ signals behave very similar to ultrasonic pulse echo signals.

\section{Emitter}

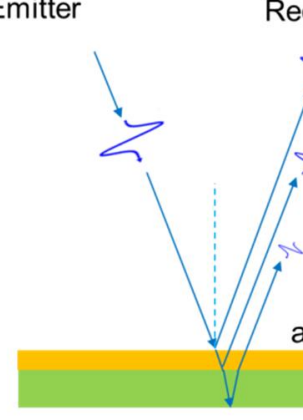

Receiver

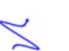

THz Pulse Signals

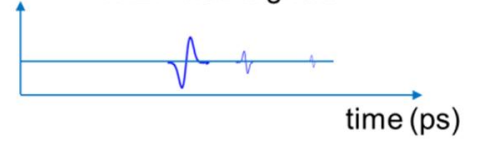

Fig. 8. Schematic drawing of the THz pulse-echo method including the reflectivity at the interfaces. The reflectivity depends on the refractive indices of the layers.

The reflection and transmission of $\mathrm{THz}$ waves at an interface between two media can be calculated with Eqs. (8) and (9) for perpendicularly incident waves.

$$
\begin{aligned}
& r=\frac{n_{1}-n_{2}}{n_{1}+n_{2}}=\frac{n-1}{n+1} \\
& t=\frac{2 n_{1}}{n_{1}+n_{2}}=\frac{2}{n+1}
\end{aligned}
$$

In case of an angle between $\mathrm{THz}$ emitter and receiver, the Fresnel relations need to be considered as well as the polarization effect. Since the coating thickness of the sample is in the sub-millimetre range, the angle effect is very small and can be neglected for the thickness determination.

The time required for the $\mathrm{THz}$ pulse to propagate through the coating and to be reflected back to the receiver can be determined with Eq. (10), where $c$ is the speed of the light, $d$ the coating thickness, $n$ the refractive index respectively.

$$
2 d=c \frac{\Delta \mathrm{t}}{n}
$$

\subsection{Experimental results}

Fig. 9 shows the measurement setup with the 2D scan stage for the $\mathrm{THz}$ imaging. The imaging software is applied for the data analysis such as time delay map, Fourier spectroscopy of the signal and contrast enhancement algorithms. The measurement results are shown in Fig. 10.

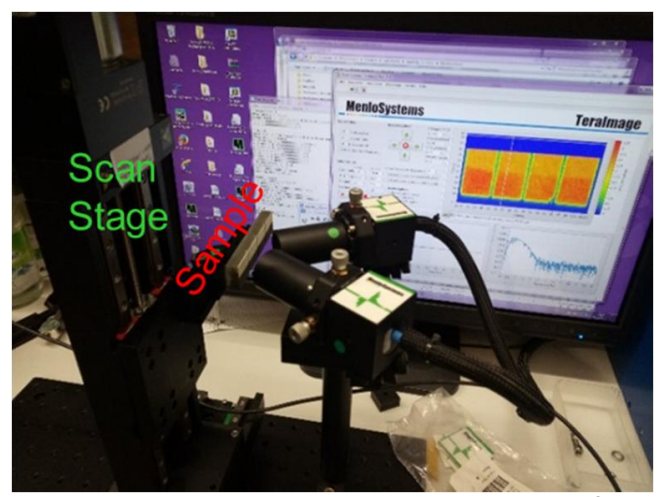

Fig. 9. Measurement setup (reflection measurement) with the $2 D$-scan device for $T H z$ imaging of the zirconia coated IN 738 sample.

In Fig. 10 the scan image with a line profile shows the time delay profile, where the time delay between the first and the second reflection is coded with a colour scale (Fig. 10 left). The numbers above the scan images are the thickness values which are set in the manufacturing process. The time delays between the first and the second reflection are shown in Fig. 10 on the right, whereas the measurement positions 1 to 4, corresponding to the 4 steps of the sample, are marked on the time delay image. The $\mathrm{THz}$ time delay signals show a quite large time difference (around $2.77 \mathrm{ps)}$ 
between step 2 and step 3. The time delay differences are around 1 ps between step 1 and step 2 as well as between step 3 and step 4.

Process setting values $\mu \mathrm{m}: 152.4 \quad 177.8 \quad 228.6 \quad 279.4$
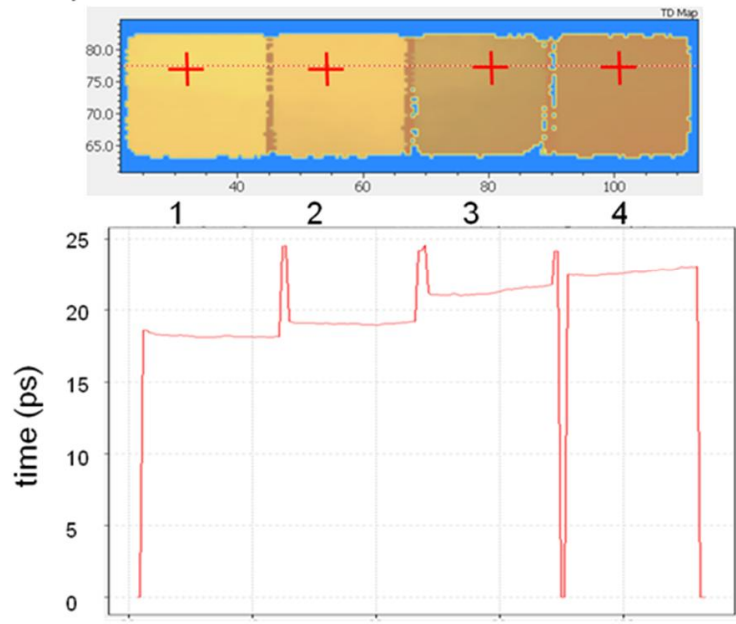

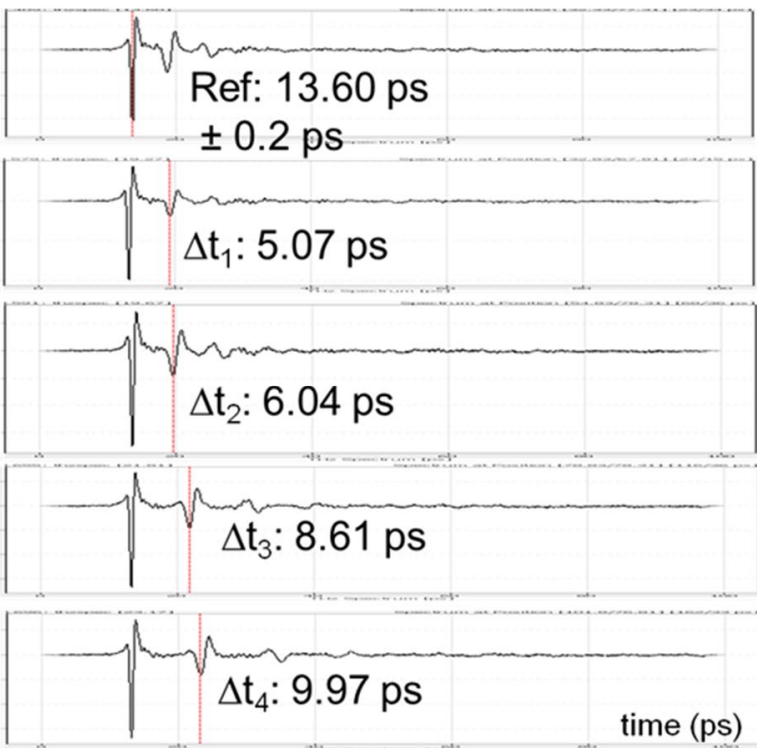

Fig. 10. Time delay map (upper left) with time delay profile (lower left) as well as time delay differences corresponding to coating thicknesses (right).

For the absolute thickness determination, a kind of measurement calibration needs to be considered. The typical refractive index of plasma sprayed ceramic coatings for $\mathrm{THz}$ signals ranges from 4.5 to 5 [26]. For 1 ps time difference between two reflections, the coating thickness may vary from $30 \mu \mathrm{m}$ to $33 \mu \mathrm{m}$. With Eq. (10), the coating thicknesses can be estimated. In Table 4 the four coating steps are estimated with an assumption for the refractive index $(n=4.5-5.0)$ in the $\mathrm{THz}$ range.

Table 4. Extracted and calculated values for the THz-TDS imaging.

\begin{tabular}{|c|c|c|c|c|}
\hline & Step 1 & Step 2 & Step 3 & Step 4 \\
\hline Nominal TBC thickness ( $\mu \mathrm{m})$ & 152.40 & 177.80 & 228.60 & 279.40 \\
\hline$\Delta t(p s) \pm 0.2(p s)$ & 5.07 & 6.04 & 8.61 & 9.97 \\
\hline $\begin{array}{l}\text { TBC thickness with THz-TDS } \\
\text { for } n=4.5-5.0\end{array}$ & $152.1-169.0$ & $181.2-201.3$ & $258.3-287.0$ & $299.1-332.3$ \\
\hline
\end{tabular}

The temporal THz signals in Fig. 11 show the surface reflection and interface reflections at step 1 and step 4 , whereas the $\mathrm{THz}$ spectrum of signals at step 1 and step 4 show the damping effect up to $0.5 \mathrm{THz}$ due to the thicker coating layer at step 4.
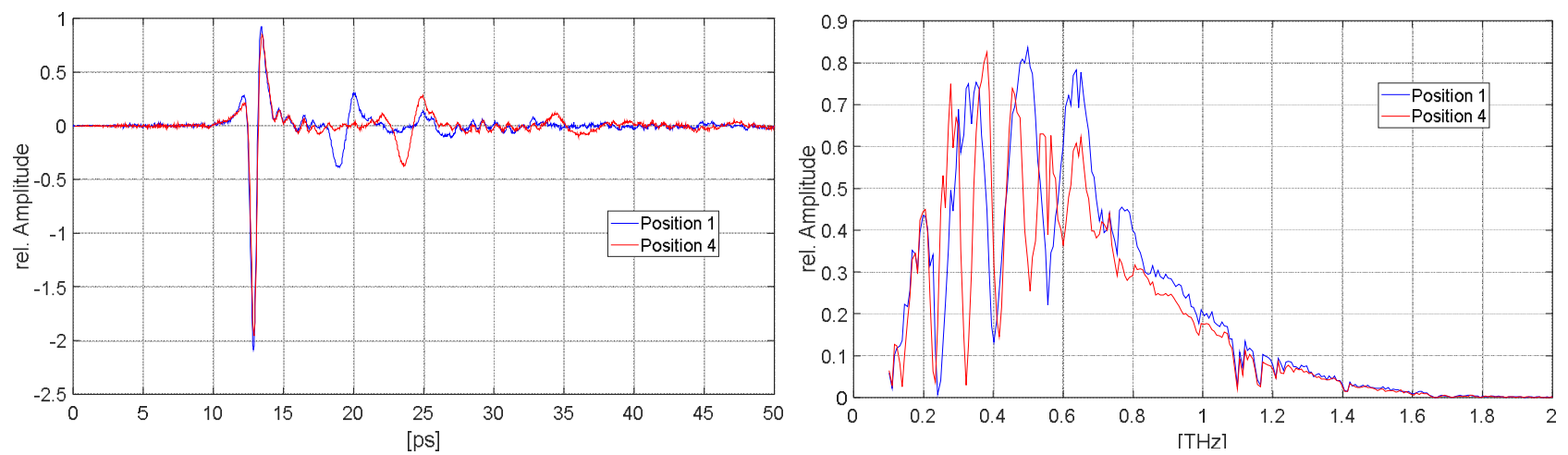

Fig. 11. Temporal THz signal (left) and the corresponding spectrum of the zirconia coating on IN 738 (right).

For the pulse-thermography the ceramic coating surface was coated with a thin graphite layer to increase emissivity and avoid depth absorption of ceramic coatings. This thin graphite layer has a strong impact on the $\mathrm{THz}$ 
measurements due to high refractive index of the graphite layer. The graphite layer causes an additional time difference of around 1 ps. For pulse-thermography this thin graphite layer has much less effect on the thickness measurements.

\section{Discussion and comparison}

Table 5 shows the compiled values of the TBC layer which resulted from the measurements with pulsethermography and THz-TDS imaging. Also included is again the standard deviation for each averaged value. Since the nominal thickness cannot be taken as a reference because the process is hard to control precisely, additionally to the two methods of thickness evaluation discussed here, the method of eddy current testing was employed. Cutting and microscopic examination could not be performed without damaging the sample. A test with computed tomography did not yield results because of the density of IN 738 and therefore problems with the radiolucency.

Table 5. Compilation of the nominal, extracted and measured properties of the TBC layer. For the error margins the standard deviation of the averaged values was used.

\begin{tabular}{|l|l|l|l|l|}
\hline & Step 1 & Step 2 & Step 3 & Step 4 \\
\hline $\begin{array}{l}\text { Averaged TBC thermal } \\
\text { conductivity }(\mathbf{W} /(\mathbf{m K}) \text { ) } \\
\text { with numerical flash model }\end{array}$ & $0.331 \pm 0.011$ & $0.370 \pm 0.007$ & $0.443 \pm 0.034$ & $0.546 \pm 0.021$ \\
\hline Nominal TBC thickness $(\boldsymbol{\mu m})$ & 152.4 & 177.8 & 228.6 & 279.4 \\
\hline $\begin{array}{l}\text { Averaged TBC thickness }(\boldsymbol{\mu m}) \\
\text { measured with eddy current }\end{array}$ & $249.3 \pm 6.9$ & $264.1 \pm 5.5$ & $351.0 \pm 7.1$ & $391.6 \pm 13.4$ \\
\hline $\begin{array}{l}\text { Averaged TBC thickness }(\boldsymbol{\mu m}) \\
\text { with numerical flash model }\end{array}$ & $116.3 \pm 2.5$ & $145.3 \pm 3.4$ & $221.9 \pm 7.7$ & $274.8 \pm 2.9$ \\
\hline $\begin{array}{l}\text { TBC thickness with THz-TDS } \\
\text { for } \boldsymbol{n}=\mathbf{4 . 5}-\mathbf{5 . 0}\end{array}$ & $152.1-169.0$ & $181.2-201.3$ & $258.3-287.0$ & $299.1-332.3$ \\
\hline
\end{tabular}

It is noticeable that the thermal conductivity is not constant for different thicknesses. For thicker TBC coating, higher thermal conductivity can also be observed. The difference between steps also appears to increase with the thickness. This could mean that the crystallisation process or rather the crystal structure changes with the thickness to allow better conduction.

There is a variation of up to 115 percent between the determined thicknesses for all of the employed techniques (Namely, the largest difference is between thermography and eddy current testing). One problem is that the used methods measure thickness differently. While thermography and $\mathrm{THz}$ measure roughly the median of the surface roughness, eddy current determines the thickness by using the outermost edge of the surface roughness. This means, due to the measurement principle, eddy current will always measure a larger thickness than the other two methods. Still, the difference is unusually large with $120-130 \mu \mathrm{m}$ to thermography and 100-60 $\mu \mathrm{m}$ to $\mathrm{THz}$, which would mean up to 60 $\mu \mathrm{m}$ surface roughness on the inner and outer surface of the TBC. One possible explanation could be the formation of a thermally grown oxide in between the TBC and the BC which could be part of the measured thickness with eddy current but not with $\mathrm{THz}$ and pulse-thermography.

Analogously to THz, pulse-thermography also sees the largest increase in thickness between step 2 and step 3 . While THz results show a thicker TBC layer than the nominal one, thermography always sizes a thinner layer. Especially for step 1 and 2, thermography determines a value with a difference of about $40 \mu \mathrm{m}$ to the value with THz-TDS. Since the refraction index of coatings plays an important role in thickness measurements with $\mathrm{THz}$, the used literature values can differ from the real case. Measurements concerning the refraction index of ceramic coatings in the THz region will be continued. To definitely clarify the differences between the employed techniques, further investigations on comparable systems are needed.

\section{Conclusion}

We examined an Inconel 738 sample coated with an yttria-stabilised zirconia two-layered thermal barrier coating system in four steps of different thickness with pulse-thermography and terahertz time domain spectroscopy imaging. Both techniques are able to identify the thickness of the coating. THz-TDS can also evaluate the THz spectrum of the coating whereas thermography is able to determine its thermal conductivity.

While thermography needs the help of a numerical model and nonlinear regression fitting as well as a lot of input parameters for the different layers in a TBC system, THz-TDS imaging needs a calibration via the reflective index of the coating material in the $\mathrm{THz}$ range. Furthermore, thermography needs an additional graphite coating due to low emissivity and partial transparency of YSZ. THz-TDS imaging on the other hand, can operate (only) without this coating. 


\section{REFERENCES}

[1] ASTM E1461-07, "Standard Test Method for Thermal Diffusivity by the Flash Method," ASTM International, West Conshohocken, PA, 2007.

[2] W. J. Parker, R. J. Jenkins, C. P. Butler and G. L. Abbott, "Flash method of determining thermal diffusivity, heat capacity, and thermal conductivity," J. Appl. Phys., vol. 32, no. 9, pp. 1679-1684, 1961.

[3] T. Bennett and F. Yu, "A nondestructive technique for determining thermal properties of thermal barrier coatings," $J$. Appl. Phys., vol. 97, no. 1, p. 013520, 2005.

[4] P. Bison, F. Cernuschi and E. Grinzato, "In-depth and in-plane thermal diffusivity measurements of thermal barrier coatings by IR camera: Evaluation of ageing," Int. J. Thermophys., vol. 29, no. 6, pp. 2149-2161, 2008.

[5] D. L. Balageas, J.-C. Krapez and P. Cielo, "Pulsed photothermal modeling of layered materials," J. Appl. Phys., vol. 59, no. 2, pp. 348-357, 1986.

[6] J. G. Sun, "Pulsed thermal imaging measurement of thermal properties for thermal barrier coatings based on a multilayer heat transfer model," J. Heat Transfer, vol. 136, no. 8, p. 081601, August 2014.

[7] L. Sripragash, M. Goldammer and M. Kördel, "Numerical modeling and comparison of flash thermographic response," in Proceedings of the Thermographie-Kolloquium 2017, Berlin, 2017.

[8] D. Fratzscher, J. Beckmann, L. S. Von Chranowski and U. Ewert, "Computed THz-Tomography," 18th World Conference on Nondestructive Testing, Durban, South Africa, 16-20 April 2012.

[9] J. Jonuscheit, "Terhertz Waves for Thickness Analyses. Non-destructive component analysis using terahertz time domain spectroscopy," Optik \& Photonik, vol. 11, no. 1, pp. 30-33, 18 February 2016.

[10] J. Dong, B. Kim, A. Locquet, P. D. N. McKeon and D. S. Citrin, "Nondestructive evaluation of forced delamination in glass fiber-reinforced composites by terahertz and ultrasonic waves," Composites Part B: Engineering, vol. 79, pp. 667-675, 15 September 2015.

[11] F. Ospald, W. Zouaghi, R. Beigang, C. Matheis, J. Jonuscheit, B. Recur, J.-P. Guillet, P. Mounaix, W. Vleugels, P. V. Bosom, L. V. González, I. López, R. M. Edo, Y. Sternberg and M. Vandewal, "Aeronautics composite material inspection with a terahertz time-domain spectroscopy system," Optical Engineering, vol. 53, no. 3, 16 Dezembre 2013.

[12] J. G. Sun, "Thermal imaging measurement accuracy for thermal properties of thermal barrier coatings," Ceram. Eng. Sci. Proc., vol. 32, no. 3, pp. 15-22, 2011.

[13] A. C. Tam and B. Sullivan, "Remote sensing applications of pulsed photothermal radiometry," Appl. Phys. Lett., vol. 43, no. 4, pp. 333-335, May 1983.

[14] W. P. Leung and A. C. Tam, "Thermal diffusivity in thin films measured by noncontact single-ended pulsed-laserinduced thermal radiometry," Optivs letters, vol. 9, no. 3, pp. 93-95, 1984.

[15] W. Leung and A. C. Tam, "Techniques of flash radiometry," J. Appl. Phys., vol. 56, no. 1, pp. 153-161, 1984.

[16] D. Greenspan, Ed., Discreet numerical methods in physics and engineering, 1. ed., vol. 107, New York: Academic Press, 1974.

[17] S. M. Shepard, L. J. R., B. A. Rubadeux, D. Wang and A. T., "Reconstruction and enhancement of active thermographix image sequences," Opt. Eng., vol. 42, no. 5, pp. 1337-1342, May 2003.

[18] The international nickel company, INC., "Alloy IN-738. Technical Data," [Online]. Available: http://www.nipera.org/ /Media/Files/TechnicalLiterature/IN_738Alloy_PreliminaryData_497_.pdf. [Accessed 09 March 2018].

[19] K. E. Peiponen, A. Zeitler and M. Kuwata-Gonokami, Terahertz Spectroscopy and Imaging, 1. ed., Berlin Heidelberg: Springer-Verlag, 2012.

[20] S. Perkowitz and G. Busse, "Far-infrared optoacoustic material probing and imaging," Opt. Lett., vol. 5, no. 6, pp. 228-229, 1980.

[21] S. Kojima, M. Wada Takeda and S. Nishizawa, "Terahertz time domain spectroscopy of complex dielectric constants of boson peaks," Journal of Molecular Structure, Vols. 651-653, pp. 285-288, 1 June 2003.

[22] W. Zouaghi, M. D. Thomson, K. Rabia, R. Hahn, V. Blank and H. G. Roskos, "Broadband terahertz spectroscopy: principles, fundamental research and potential for industrial applications," Eur. J. Phys., vol. 34, no. 6, p. 179, 22 October 2013.

[23] Menlo Systems GmbH, THz-TDS System Selection Guide. Finding the right solution for THz applications, Martinsried, 2017.

[24] T. Hochrein, R. Wilk, M. Mei, R. Holzwarth, N. Krumbholz and M. Koch, "Optical sampling by laser cavity tuning," Optics Express, vol. 18, no. 2, pp. 1613-1617, 18 January 2010.

[25] D. R. Clarke, M. Oechsner and N. P. Padture, "Thermal barrier coatings for more efficient gas turbine engines," Materials Research Society Bulletin, vol. 37, no. 10, pp. 891-898, 1 October 2012.

[26] T. Fukuchi, N. Fuse, M. Mizuno and K. Fukunaga, "THz Measurement of Refractive Index and Thickness of Ceramic Coating on a Metal Substrate," 2013 Conference on Lasers and Electro-Oprics Pacific Rim, Kyoto, Japan, 30 June 4 July 2013, 2013. 